\title{
The early protective innate immune response against West Nile virus \\ Stephane Daffis ${ }^{1}$, Melanie A Samuel ${ }^{1}$, Mehul S Suthar ${ }^{2}$, Brian K Keller ${ }^{2}$, Michael Gale $\mathrm{Jr}^{2}$ and Michael S Diamond*1
}

Address: ${ }^{1}$ Washington University School of Medicine, St Louis, MO, USA and ${ }^{2}$ University of Washington School of Medicine, Seattle, WA, USA

Email: Michael S Diamond* - diamond@borcim.wustl.edu

* Corresponding author

from Infectious diseases of the nervous system: pathogenesis and worldwide impact

Paris, France. 10-13 September 2008

Published: 23 September 2008

BMC Proceedings 2008, 2(Suppl I):S8

This abstract is available from: http://www.biomedcentral.com/1753-656I/2/SI/S8

(c) 2008 Daffis et al; licensee BioMed Central Ltd.

The type I interferon (IFN)- $\alpha / \beta$ response plays an essential role in controlling West Nile virus (WNV) tropism, replication and spread to the central nervous system (CNS) in mice. Induction of IFN $\alpha / \beta$ relies on the detection of viruses by specific host factors known as pattern-recognition receptors. Central to this signaling pathway are the transcription factors interferon regulatory factors (IRF)-3 and IRF-7, which induce IFN- $\alpha / \beta$ gene expression. We used IRF-3-/-, IRF-7-/- and IRF-3-/- $\times$ IRF-7-/- mice to define how a deficiency of master innate immune response transcriptional factors impacts WNV pathogenesis and IFN signaling responses. Whereas WNV-infected wild type mice exhibited 35\% mortality, mice lacking IRF3 and/or IRF-7 had 100\% mortality with enhanced viral burdens in peripheral and CNS organs, with altered tissue and cellular tropism. A deficiency in IRF-3 did not alter the systemic IFN response in vivo or IFN- $\alpha / \beta$ production ex vivo in immune cells. However, IRF-3 restricted WNV replication in macrophages by regulating basal expression of host defense molecules. Moreover, IRF-3 regulated WNV replication through IFN-dependent mechanisms in neurons. In contrast, an absence of IRF-7 abolished the systemic IFN production in mice and abrogated IFN- $\alpha$ responses in primary cells with little effect on IFN- $\beta$ induction. Notably, a combined deficiency of IRF-3 and IRF-7 sustained enhanced WNV replication in vivo and in primary cells but did not completely abolish the IFN-b responses. These data show an essential role of IRF-3 and IRF-7 in the early control of WNV infection by regulating not only IFN responses but also antiviral programs in a cell/tissue-specific manner. 